Interfaces and Free Boundaries 1, (1999) 81-105

\title{
Energies for incoherent films: an analytical approach
}

\author{
PAOLO CERMelli \\ Dipartimento di Matematica, Università di Torino, Italy \\ MORTON E. GURTIN \\ Department of Mathematical Sciences, Carnegie-Mellon University, Pittsburgh, PA, USA \\ AND \\ GIOVANNI LEONI \\ Dipartimento di Scienze e Tecnologie Avanzate, Università del Piemonte Orientale, \\ Alessandria, Italy
}

[Received 21 December 1998 and in revised form 23 January 1999]

\begin{abstract}
This work discusses the role of interfacial energy for problems involving an epitaxial layer on a rigid substrate. Using the calculus of variations, resulting microstructures are determined for a large class of interfacial energies; the qualitative features of these microstructures demonstrate a strong dependence on the smoothness and convexity of the energy. This work is meant to provide insight into deciding appropriate energies for a large class of incoherent interfaces.
\end{abstract}

\section{Introduction}

At an interface between crystalline solids, two opposing mechanisms compete to determine the resulting structure (see e.g. the review article by Matthews [19]). The minimum-energy configuration of the bulk material occurs at the stress-free state for each solid. But when the lattice parameters of the two materials differ, complete relaxation to bulk equilibrium would result in a crystalline structure that is discontinuous at the interface. On the other hand, the interface reaches its minimum-energy configuration when there is an exact matching of the atoms of the two solids across the interface. This state of perfect coherence is tenable, provided that the stresses due to the deviation from equilibrium of the bulk material are not too strong. But there is a threshold at which these stresses are too severe to support a coherent interface, and the structure of the interface undergoes dramatic changes: dislocations appear that relax the bulk stresses, and an extreme situation may be reached in which all regularity of the atomic bonding at the interface is lost.

A proper choice of interfacial energy is crucial in describing the competition discussed above, but a chief difficulty in deciding on such an energy is the extreme range of behaviour it must embody, since it must characterize: (i) perfect matching of the atoms at the interface; (ii) dislocations distributed in a somewhat regular manner; and (iii) situations in which the abutting lattices are completely mismatched.

Specific interfacial energies have been proposed, some based on microscopic calculations (van der Merwe [21, 22, 23], du Plessis \& van der Merwe [9], Fletcher \& Adamson [11], Fletcher [10]) and others on phenomenological considerations (Leo \& Hu [18]), and it might be useful to have at hand a catalogue of possible energy functions together with an analysis of their predictions. From a qualitative perspective, such a catalogue is well within the scope of modern variational analysis. 
Indeed, while it may be difficult to determine the actual bulk energy for an elastic solid undergoing small strains, it is not difficult to show that, whatever form the actual energy should take, it should generally be a strictly convex function of strain. Here our goal is to develop similar crude qualitative results for interfaces.

To isolate the underlying physics, we keep the geometry as simple as possible by restricting attention to a flat epitaxial layer on a rigid semi-infinite substrate, with the thickness $h$ of the layer used to modulate the contribution of bulk energy, which we consider to be quadratic.

We further restrict attention to plane strain as described by Cartesian coordinates $(x, y)$, with interface the line $y=0$ and layer defined by $0 \leqslant y \leqslant h$.

The three basic behavioural patterns a simplified model should describe might be summarized as follows [19]:

(i) for sufficiently thin layers, interfacial energy should prevail over bulk energy, and the interface should remain coherent;

(ii) above a threshold value for the layer-thickness $h$, dislocations should migrate to the interface to relieve the high local stresses, and the relative displacement between phases should develop discontinuities, although portions of the interface might remain coherent;

(iii) for sufficiently large $h$ and for solids whose stress-free states are sufficiently disparate, equilibrium should result in an extreme loss of regularity consistent with a complete mismatch of the abutting lattices.

We measure the displacement from a configuration of the film in which the lattices of the film and the substrate are perfectly matched; such a configuration would generally correspond to a stressed state for the film. Since the substrate is rigid, if we assume that the layer does not separate from the substrate, then the $x$-component $u$ of the displacement of the film at the interface measures the relative displacement between film and substrate. We refer to the tangential derivative $\gamma=u_{x}$ of this displacement as the incoherency strain. When $\gamma$ vanishes on an open set, the relative displacement is constant, and the lattice structures are viewed as perfectly matched. Conversely, when $\gamma$ is not constant, the lattices are deformed relative to one another, and $\gamma$ is a measure of the mismatch. Finally, when the relative displacement is discontinuous, so that $\gamma$ is unbounded, dislocations concentrate over very small regions, namely the set at which the relative displacement jumps.

Following common practice (e.g. Matthews [19]), we refer to an interface as coherent when the displacement is constant (so that $\gamma=0$ ) and smoothly incoherent when $\gamma$ is smooth and non-vanishing. More interesting are the intermediate cases, which correspond to equilibrium states that can be realized as limits of sequences whose elements describe a fine mixture of coherent and incoherent regions; we call such interfaces finely incoherent. This terminology is somewhat imprecise, since we shall encounter two types of finely incoherent interfaces: one for which $\gamma$ becomes unbounded, so that dislocations concentrate over small regions, and the other for which $\gamma$ oscillates between two fixed values and hence describes a mixture of smoothly incoherent patches.

We restrict attention to interfacial energies that depend only on $\gamma$ (e.g. Larché \& Cahn [17], Cahn \& Larché [4], Cermelli \& Gurtin [5,6], Leo \& Hu[18]). We study four classes of energy functions: (i) smooth and convex; (ii) nonsmooth but convex; (iii) nonsmooth and nonconvex; (iv) nonsmooth and concave.

The central effects - namely fine or smooth incoherency and the existence of a threshold for incoherency-depend on whether or not the energy is convex and whether or not the energy is smooth. Nonconvex energies yield finely incoherent interfaces; energies whose convex envelope is 
nonsmooth exhibit a threshold effect.

Our specific results may be described as follows. Consider first a smooth and convex energy $f(\gamma)$ (Fig. 1), for instance quadratic in $\gamma$. Then our analysis shows that the interface is always smoothly incoherent, and there is no threshold for incoherency.

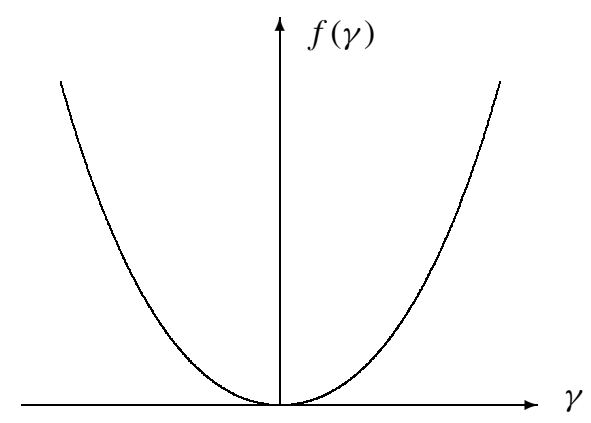

FIG. 1. Smooth convex energy $f$.

For convex energies that are not smooth at $\gamma=0$ (Fig. 2) the interface remains coherent for small thicknesses, but the equilibrium state is one of smooth incoherency when the threshold thickness is exceeded. Such energies might be appropriate to systems with large misfit.

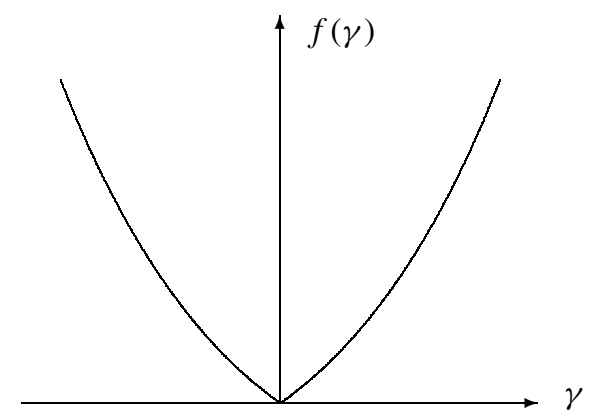

FIG. 2. Nonsmooth convex energy $f$.

A more interesting type of behaviour, for an interfacial energy that is not smooth at $\gamma=0$ (Fig. 3), occurs when the energy is concave for small values of $\gamma$ but ultimately convex for large values. Such an energy was proposed by Leo \& Hu [18] and results in an interface that, although coherent for small thicknesses, becomes finely incoherent above a threshold, with the infimum of the energy realized by sequences corresponding to fine mixtures of coherent and incoherent patches. A drawback of this choice of energy is its special form: it is necessary to assign in advance, as a constitutive parameter, the incoherency strain that determines the mixture.

A possible improvement of this model, motivated by the necessity of modelling interfaces between materials with large misfits, is based on an interfacial energy function that is concave almost everywhere, but with non-smooth local minima at definite values of the incoherency strain, 

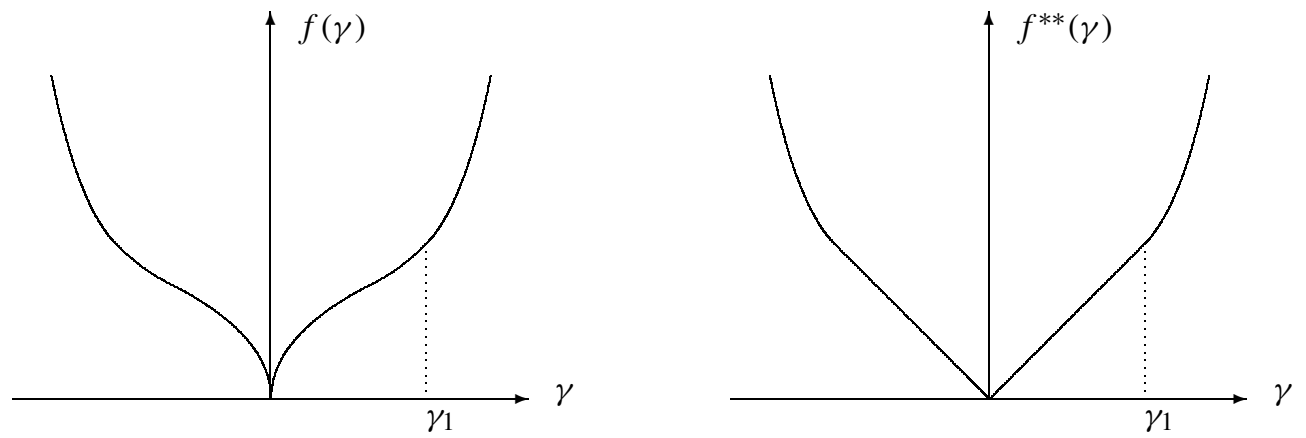

FIG. 3. Nonsmooth nonconvex energy $f$ and its convex envelope $f^{* *}$.

which, when connected, convexify the energy (Fig. 4). As the thickness $h$ of the layer increases, the interface changes from coherency to fine incoherency, and then to smooth incoherency with an incoherency strain corresponding to one of these minima. Also, there are intervals of $h$ for which the interface is either coherent or smoothly incoherent: a threshold effect thus shows up also in the transition to and from smooth incoherency, since the lattices try to stay 'glued together' at definite values of the incoherency strain (cf. the notion of coincidence boundary in [19]).
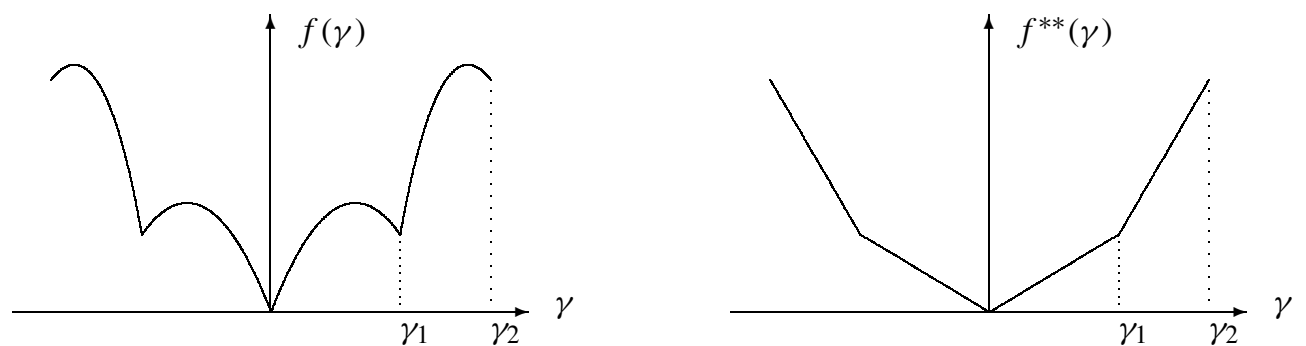

FIG. 4. Nonsmooth scalloped energy $f$ and its convex envelope $f^{* *}$.

Finally, we consider concave energies that are not smooth at zero (Fig. 5), but are otherwise smooth and concave (and may be thus considered as a special case of the preceding ones). Such energies are motivated by the original treatments of incoherent interfaces based on molecular theories [21,22, 23,9,10,11]. These energies have the most interesting behaviour: in addition to displaying a threshold effect for the transition to incoherency, they model finely incoherent interfaces that contain dislocations, i.e. for which the minimizing sequences are characterized by unbounded relative strains. These energies might reasonably describe small-misfit epitaxial layers.

As the goal of this study is a qualitative comparison of a variety of interfacial energies, we do not allow for a redistribution of the material within the film.

The mathematical techniques we use in this paper are based on classical results of the calculus of variations, and the general approach follows closely ideas developed by Leo \& $\mathrm{Hu}$ [18] for the 

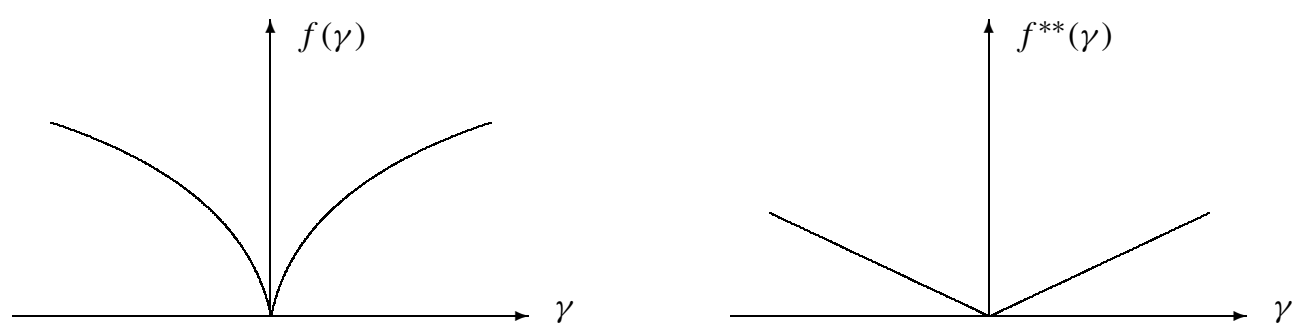

FIG. 5. Nonsmooth concave energy $f$ and its convex envelope $f^{* *}$.

interfacial energy function displayed in Fig. 3. We essentially compute the minima of the total energy functional (bulk and interfacial energies), and then analyse the corresponding minimizing sequences.

We are currently working to extend the results to non-quadratic bulk energies and curved interfaces.

\section{Statement of the problem}

Our model describes the equilibrium of an epitaxial layer on a rigid substrate. Assuming that the layer has height $h$, but is infinite in the other directions, we study a model problem for a plane section of the film, within the context of plane elasticity.

We assume that the layer occupies the infinite strip $\mathbb{R} \times[0, h] \subset \mathbb{R}^{2}$. We let $(x, y)$ denote cartesian coordinates in $\mathbb{R}^{2}$ with $x \in \mathbb{R}$ and $y \in[0, h]$, and we write $\mathbf{i}=(1,0)$ and $\mathbf{j}=(0,1)$. We limit our discussion to plane displacements $\mathbf{u}(x, y)$ of the layer and to situations in which $\mathbf{u}(x, y)$ is periodic in $x$. With this in mind, we divide the strip into cells of unit length, write $\Omega=[0,1] \times[0, h]$ for a typical cell, and restrict attention to behaviour in $\Omega$. Periodicity then requires that $\mathbf{u}(1, y)-$ $\mathbf{u}(0, y)$ be constant (which, by (2.1), is equivalent to the requirement that the strain $\mathbf{E}$, defined below, satisfy $\mathbf{E}(1, y)-\mathbf{E}(0, y)=$ constant $)$. We assume that the layer cannot separate from the substrate; thus, since $y=0$ defines the interface between the layer and the substrate,

$$
\mathbf{u}(x, 0) \cdot \mathbf{j}=0 \quad x \in[0,1],
$$

and the periodicity condition takes the stronger form

$$
\mathbf{u}(1, y)=\mathbf{u}(0, y)+(\text { const. }) \mathbf{i} \quad y \in[0, h] .
$$

Let

$$
u(x, y):=\mathbf{u}(x, y) \cdot \mathbf{i} ;
$$

we define the incoherency strain $\gamma(x)$ by

$$
\gamma(x):=u_{x}(x, 0)
$$

and refer to the interface as coherent if

$$
\gamma(x)=0 \quad x \in[0,1],
$$


and incoherent otherwise.

We work within the theory of small deformations, so that

$$
\mathbf{E}=\frac{1}{2}\left(\nabla \mathbf{u}+\nabla \mathbf{u}^{\top}\right)
$$

represents the strain in the layer. Note that $\gamma=\mathbf{i} \cdot \mathbf{E}(x, 0) \mathbf{i}$. The displacement is measured from a configuration of the layer in which the lattices of the film and the layer are perfectly matched; this configuration, in which $\mathbf{E}=0$, will not correspond to a minimum-energy state of the film, which we assume to occur at a strain $\mathbf{E}_{0}$. We assume that this mismatch strain has the specific form

$$
\mathbf{E}_{0}=e_{0} \mathbf{i} \otimes \mathbf{i}
$$

with $e_{0}>0$. Specifically, we assume that the layer is composed of a homogeneous isotropic elastic material with positive-definite, quadratic strain energy $w$. Thus, bearing in mind the mismatch,

$$
w(\mathbf{A})=\frac{1}{2} \lambda[\operatorname{tr}(\mathbf{A})]^{2}+\mu\left[\operatorname{tr}\left(\mathbf{A}^{2}\right)\right], \quad \mathbf{A}=\mathbf{E}-\mathbf{E}_{0},
$$

with Lamè moduli $\mu$ and $\lambda$ constant and such that $\mu>0,2 \mu+3 \lambda>0$.

We assume that the interfacial energy is a function $f(\gamma)$ of the incoherency strain. Precisely, we assume that

$$
f: \mathbb{R} \rightarrow[0, \infty), \quad f \text { is even and continuous, } \quad f(\gamma)>0 \text { for } \quad \gamma \neq 0,
$$

and that $f$ satisfies the growth condition

$$
f(\gamma) \leqslant C\left(1+|\gamma|^{q}\right)
$$

with $C>0$ and $q \geqslant 1$. We also assume, without loss in generality, that

$$
f(0)=0 .
$$

The total energy of the system is given by

$$
J(\mathbf{u})=\int_{\Omega} w\left(\mathbf{E}-\mathbf{E}_{0}\right) \mathrm{d} x \mathrm{~d} y+\int_{0}^{1} f(\gamma) \mathrm{d} x .
$$

In analyzing this system it is convenient to consider the bulk and interfacial energies independently, and for that reason we write

$$
F(\mathbf{u})=\int_{\Omega} w\left(\mathbf{E}-\mathbf{E}_{0}\right) \mathrm{d} x \mathrm{~d} y, \quad I(u)=\int_{0}^{1} f(\gamma) \mathrm{d} x .
$$

Here and when there is no danger of confusion, we will write $u$ for the function with values $u(x, 0)$.

In this paper we shall discuss the minimization of the functional $J(\mathbf{u})$ on the space of all sufficiently smooth vector fields $\mathbf{u}$ on $\Omega$ that satisfy the compatibility condition (2.1) and the periodicity condition (2.2) 


\section{General results}

Due to the quadratic growth condition of the bulk energy density $w$, and to (2.3), the natural setting for our problem is the space of all functions $\mathbf{u} \in W^{1,2}\left(\Omega, \mathbb{R}^{2}\right)$ whose trace $\tau \mathbf{u}$ (in the sense of Sobolev spaces) belongs to $W^{1, q}\left(\partial \Omega, \mathbb{R}^{2}\right)$. Thus, when $q>1$, we consider the fractional Sobolev space

$$
\begin{aligned}
W^{1+1 / q, q}\left(\Omega, \mathbb{R}^{2}\right):= & \left\{\mathbf{u} \in W^{1, q}\left(\Omega, \mathbb{R}^{2}\right):\right. \\
& \left.\iint_{\Omega \times \Omega} \frac{|\nabla \mathbf{u}(x, y)-\nabla \mathbf{u}(z, w)|^{q}}{|(x, y)-(z, w)|^{3}} \mathrm{~d} x \mathrm{~d} y \mathrm{~d} z \mathrm{~d} w<\infty\right\},
\end{aligned}
$$

and, when $q=1$, the classical Sobolev space $W^{2,1}\left(\Omega, \mathbb{R}^{2}\right)$. By Sobolev's embedding, for all $q \geqslant 1$,

$$
W^{1+1 / q, q}\left(\Omega, \mathbb{R}^{2}\right) \subset W^{1,2 q}\left(\Omega, \mathbb{R}^{2}\right)\left(\subset W^{1,2}\left(\Omega, \mathbb{R}^{2}\right)\right) .
$$

Moreover, for $q>1$,

$$
W^{1+1 / q, q}\left(\Omega, \mathbb{R}^{2}\right) \subset C^{0,1-1 / q}\left(\bar{\Omega}, \mathbb{R}^{2}\right),
$$

while for $q=1$, and since $\Omega$ is a rectangle (see [1], Lemma 5.8), ${ }^{*}$

$$
W^{2,1}\left(\Omega, \mathbb{R}^{2}\right) \subset C^{0}\left(\bar{\Omega}, \mathbb{R}^{2}\right) .
$$

By the trace theorem for polygons (see e.g. [15], Theorems 1.5.2.1 and 1.5.2.8) and Sobolev's embeddings, we can define the trace $\tau \mathbf{u}$ of any $\mathbf{u} \in W^{1+1 / q, q}\left(\Omega, \mathbb{R}^{2}\right)$ as a continuous function on $\partial \Omega$ such that

$$
\left.\tau \mathbf{u}\right|_{\Gamma_{j}} \in W^{1, q}\left(\Gamma_{j}, \mathbb{R}^{2}\right), \quad j=1, \ldots, 4,
$$

where $\Gamma_{j}$ are the sides of $\Omega$. In what follows, when the meaning is clear, we will denote $\tau \mathbf{u}$ simply by $\mathbf{u}$.

Letting $W$ denote the space

$$
W=\left\{\mathbf{u} \in W^{1+1 / q, q}\left(\Omega, \mathbb{R}^{2}\right): \mathbf{u} \text { satisfies }(2.1) \text { and }(2.2)\right\}
$$

we consider the following problem.

Minimization PROBlem (M) Find a displacement field $\mathbf{u} \in W$ such that

$$
J(\mathbf{u}) \leqslant J(\hat{\mathbf{u}}) \quad \text { for all } \quad \hat{\mathbf{u}} \in W .
$$

If $\mathbf{u} \in W$ is a solution of (M), then so is $\mathbf{u}+c \mathbf{i}$, for any scalar constant $c$. We may therefore assume, without loss of generality, that $u(0,0)=0$ for all $\mathbf{u} \in W$. Thus, for each fixed $\alpha \in \mathbb{R}$, consider the spaces

$$
\begin{aligned}
& W_{\alpha}=\{\mathbf{u} \in W: u(1,0)=\alpha, u(0,0)=0\}, \\
& \hat{W}_{\alpha}=\left\{u \in W^{1, q}([0,1]): u(1)=\alpha, u(0)=0\right\} .
\end{aligned}
$$

\footnotetext{
* For arbitrary Lipschitz domain we only have the weaker inclusion $W^{2,1}\left(\Omega, \mathbb{R}^{2}\right) \subset C^{0}\left(\Omega, \mathbb{R}^{2}\right) \cap L^{\infty}\left(\Omega, \mathbb{R}^{2}\right)$.
} 
Then $W_{\alpha}$ and $\hat{W}_{\alpha}$ are convex, and each $\mathbf{u} \in W_{\alpha}$ satisfies

$$
\begin{array}{lc}
\mathbf{u}(1, y)=\mathbf{u}(0, y)+\alpha \mathbf{i}, & y \in[0, h], \\
\mathbf{u}(x, 0) \cdot \mathbf{j}=0, & x \in[0,1] .
\end{array}
$$

Thus, since each cell has unit length, the subscript $\alpha$ represents the average value of the incoherency strain $\gamma=u_{x}$ corresponding to any $\mathbf{u} \in W_{\alpha}$.

Our first step in attacking problem (M) is to determine the infimum of the functional $J$. We accomplish this by first computing

$$
\inf _{\mathbf{u} \in W_{\alpha}} J(\mathbf{u})
$$

and then minimizing over all $\alpha \in \mathbb{R}$. To facilitate this, we introduce the convex envelope $f^{* *}$ of $f$ defined by

$$
f^{* *}=\sup \{g \leqslant f: g \text { convex }\} .
$$

THEOEREM ON THE INFIMIUM OF $J$. For any $\alpha \in \mathbb{R}$,

$$
g_{\text {blk }}(\alpha):=\min _{\mathbf{u} \in W_{\alpha}} F(\mathbf{u})=\frac{E h}{2\left(1-v^{2}\right)}\left(\alpha-e_{0}\right)^{2}
$$

and

$$
g_{\text {int }}(\alpha):=\inf _{u \in \hat{W}_{\alpha}} I(u)=f^{* *}(\alpha)
$$

with $E=\frac{\mu(3 \lambda+2 \mu)}{\lambda+\mu}$ Young's modulus and $v=\frac{\lambda}{2(\lambda+\mu)}$ Poisson's ratio. Moreover,

$$
\inf _{\mathbf{u} \in W} J(\mathbf{u})=\min _{\alpha \in \mathbb{R}} g(\alpha), \quad \text { where } \quad g(\alpha):=g_{\text {blk }}(\alpha)+g_{\text {int }}(\alpha) .
$$

Since the function $g$ is strictly convex, for fixed $h$ and $e_{0}$, there exists a unique solution $\alpha_{\min }$ such that

$$
g\left(\alpha_{\min }\right)=\min _{\alpha \in \mathbb{R}} g(\alpha)
$$

We will refer to $\alpha_{\min }$ as the average incoherency strain corresponding to the infimum of $J$. By the assumptions on $f$,

$$
\alpha_{\min } \in\left[0, e_{0}\right]
$$

Moreover (see e.g. [20])

$$
\begin{aligned}
0 \in \partial g\left(\alpha_{\min }\right)=\left[g_{-}^{\prime}\left(\alpha_{\min }\right), g_{+}^{\prime}\left(\alpha_{\min }\right)\right] \\
\quad=\left[\frac{E h}{1-v^{2}}\left(\alpha_{\min }-e_{0}\right)+\left(f^{* *}\right)_{-}^{\prime}\left(\alpha_{\min }\right), \frac{E h}{1-v^{2}}\left(\alpha_{\min }-e_{0}\right)+\left(f^{* *}\right)_{+}^{\prime}\left(\alpha_{\min }\right)\right],
\end{aligned}
$$

where $\partial g, g_{-}^{\prime}$, and $g_{+}^{\prime}$ are respectively the subdifferential and the left and right derivatives of the convex function $g$. As a direct corollary of the previous theorem, we have the following result. 
EXISTENCE THEOREM. (i) The minimization problem $(M)$ has a solution if and only if

$$
f^{* *}\left(\alpha_{\min }\right)=f\left(\alpha_{\min }\right)
$$

(ii) The minimization problem $(M)$ admits a solution with a coherent interface if and only if

$$
0 \leqslant h \leqslant h_{c}, \quad \text { where } \quad h_{c}:=\frac{1-v^{2}}{E} \frac{\left(f^{* *}\right)_{+}^{\prime}(0)}{e_{0}} .
$$

When (3.7) holds, then $\alpha_{\min }=0$ and (3.6) follows from the fact that $f(0)=f^{* *}(0)=0$. Thus the non-smoothness of $f^{* *}$ at zero implies the existence of a critical value $h_{c}$; for $h$ below $h_{c}$, the interface is coherent. When (3.7) fails, then $\alpha_{\min }>0$ and the interface is incoherent.

Granted incoherency, if $f^{* *}\left(\alpha_{\min }\right)=f\left(\alpha_{\min }\right)$, then the problem $(M)$ has a solution $\mathbf{u}$, and there is a well-defined incoherency strain $\gamma$ defined over the interface; in this case we will refer to the interface as being smoothly incoherent. On the other hand, for $h>h_{c}$ and $f^{* *}\left(\alpha_{\min }\right)<$ $f\left(\alpha_{\min }\right)$ (so that $\alpha_{\min }$ is not a point of interfacial stability), $(M)$ has no solution; that is, there is no function $\mathbf{u} \in W$ that minimizes $J$. In this case we will refer to the interface as finely incoherent. For such situations, even though $(M)$ has no solution, one can derive physically meaningful results by studying the properties of minimizing sequences - that is, sequences $\left\{\mathbf{u}_{n}\right\}$ with the property that $\mathbf{u}_{n} \in W$ and

$$
J\left(\mathbf{u}_{n}\right) \rightarrow \inf _{\mathbf{u} \in W} J(\mathbf{u}),
$$

or equivalently

$$
J\left(\mathbf{u}_{n}\right) \rightarrow g\left(\alpha_{\min }\right) .
$$

For any such sequence, let $\gamma_{n}(x)=\frac{\partial u_{n}(x, 0)}{\partial x}$, so that

$$
\alpha_{n}=\int_{0}^{1} \gamma_{n}(x) \mathrm{d} x
$$

is the average incoherency strain associated with $\mathbf{u}_{n}$.

We may prove the following

THEOREM ON MINIMIZING SEQUENCES. Let $\left\{\mathbf{u}_{n}\right\}$ be a minimizing sequence for the problem $(M)$ with $\alpha_{n}$ the average incoherency strain associated with $\mathbf{u}_{n}$. Then

$$
\begin{aligned}
& \alpha_{n} \rightarrow \alpha_{\min }, \\
& \mathbf{u}_{n} \rightarrow \tilde{\mathbf{u}}_{\min } \quad \text { in } \quad W^{1,2}\left(\Omega, \mathbb{R}^{2}\right),
\end{aligned}
$$

as $n \rightarrow \infty$, where

$$
\tilde{\mathbf{u}}_{\min }(x, y):=\alpha_{\min } x \mathbf{i}+\frac{v\left(e_{0}-\alpha_{\min }\right)}{(1-v)} y \mathbf{j}
$$

Thus the minimizing sequences always converge in bulk to a smooth deformation.

At the interface, although minimizing sequences may not have a classical limit when $(M)$ has no solution, the 'generalized limit', however it be visualized, corresponds to a well defined average incoherency strain, namely $\alpha_{\min }$, the incoherency strain associated with the infimum of $J$. 


\section{Proofs}

Proof. The theorem on the infimum of $J$. Fix $\mathbf{u} \in W$ and let $\alpha:=u(1,0)$. Then

$$
J(\mathbf{u})=F(\mathbf{u})+I(u(x, 0)) \geqslant \inf _{\mathbf{v} \in W_{\alpha}} F(\mathbf{v})+\inf _{v \in \hat{W}_{\alpha}} I(v) .
$$

Consider the auxiliary problem:

$\left(\mathrm{M}_{\alpha}\right)$ : minimize $F(\mathbf{u})$ on $W_{\alpha}$.

The solution of this problem exists, and is given by the displacement

$$
\tilde{\mathbf{u}}(x, y):=\alpha x \mathbf{i}+\frac{v\left(e_{0}-\alpha\right)}{(1-v)} y \mathbf{j}
$$

corresponding to the homogeneous strain

$$
\tilde{\mathbf{E}}=\alpha \mathbf{i} \otimes \mathbf{i}+\frac{v\left(e_{0}-\alpha\right)}{(1-v)} \mathbf{j} \otimes \mathbf{j}
$$

for which

$$
\min _{W_{\alpha}} F(\mathbf{u})=F(\tilde{\mathbf{u}})=\frac{E h}{2\left(1-v^{2}\right)}\left(\alpha-e_{0}\right)^{2},
$$

with $v$ Poisson's ratio and $E$ Young's modulus.

To see this, note that, for any pair of displacement fields $\mathbf{u}, \tilde{\mathbf{u}} \in W_{\alpha}$, with $\mathbf{E}$ and $\tilde{\mathbf{E}}$ the corresponding strains,

$$
w\left(\mathbf{E}-\mathbf{E}_{0}\right)-w\left(\tilde{\mathbf{E}}-\mathbf{E}_{0}\right)=w(\mathbf{E}-\tilde{\mathbf{E}})+\tilde{\mathbf{T}} \cdot(\mathbf{E}-\tilde{\mathbf{E}}),
$$

where $\tilde{\mathbf{T}}=2 \mu\left(\tilde{\mathbf{E}}-\mathbf{E}_{0}\right)+\lambda \operatorname{tr}\left(\tilde{\mathbf{E}}-\mathbf{E}_{0}\right) \mathbf{1}$ is the stress corresponding to $\tilde{\mathbf{u}}$. Thus

$$
F(\mathbf{u})-F(\tilde{\mathbf{u}})=\int_{\Omega} w(\mathbf{E}-\tilde{\mathbf{E}}) \mathrm{d} x \mathrm{~d} y+\int_{\Omega} \tilde{\mathbf{T}} \cdot(\mathbf{E}-\tilde{\mathbf{E}}) \mathrm{d} x \mathrm{~d} y .
$$

If $\tilde{\mathbf{u}}$ is sufficiently smooth to satisfy the Euler-Lagrange equation $\operatorname{div} \tilde{\mathbf{T}}=0$ in $\Omega$, and if $\tilde{\mathbf{T}}$ satisfies the natural boundary conditions $\tilde{\mathbf{T}}(x, h) \mathbf{j}=0$ for $x \in[0,1]$ and the periodicity condition $\tilde{\mathbf{T}}(0, y) \mathbf{i}=\tilde{\mathbf{T}}(1, y) \mathbf{i}$ for $y \in[0, h]$, then, since both $\mathbf{u}$ and $\tilde{\mathbf{u}}$ satisfy the conditions (3.1), we may use the divergence theorem to conclude that

$$
\int_{\Omega} \tilde{\mathbf{T}} \cdot(\mathbf{E}-\tilde{\mathbf{E}}) \mathrm{d} x \mathrm{~d} y=\int_{\Omega} \tilde{\mathbf{T}} \cdot(\nabla \mathbf{u}-\nabla \tilde{\mathbf{u}}) \mathrm{d} x \mathrm{~d} y=-\int_{0}^{1}(\mathbf{i} \cdot \tilde{\mathbf{T}}(x, 0) \mathbf{j})(u(x, 0)-\tilde{u}(x, 0)) \mathrm{d} x .
$$

Since the above requirements are met by the smooth displacement field (4.1), for which a simple computation shows that $\mathbf{i} \cdot \tilde{\mathbf{T}}(x, 0) \mathbf{j}=0$ for $x \in[0,1]$, then

$$
F(\mathbf{u}) \geqslant F(\tilde{\mathbf{u}})
$$

for any $\mathbf{u} \in W_{\alpha}$, as a consequence of the positive-definiteness of the quadratic form $w(\mathbf{E}-\tilde{\mathbf{E}})$. Thus $\tilde{\mathbf{u}}$ is a minimizer of $F$ in $W_{\alpha}$, and the corresponding value of the energy is readily computed. Further, this minimizer is unique. Suppose in fact that $\tilde{\mathbf{u}}$ and $\hat{\mathbf{u}}$ are two minimizers, so that $F(\tilde{\mathbf{u}})=F(\hat{\mathbf{u}})$ : 
then, again by the positive definiteness of the quadratic form on the right-hand side of (4.3), we have $\tilde{\mathbf{E}}=\hat{\mathbf{E}}$, and this implies that $\tilde{\mathbf{u}}=\hat{\mathbf{u}}+\mathbf{a}+b(y \mathbf{i}-x \mathbf{j})$. But $\mathbf{a}=0$ and $b=0$, since both displacements satisfy the same boundary conditions.

Moreover

$$
I(v)=\int_{0}^{1} f\left(v^{\prime}(x)\right) \mathrm{d} x \geqslant \int_{0}^{1} f^{* *}\left(v^{\prime}(x)\right) \mathrm{d} x \geqslant f^{* *}\left(\int_{0}^{1} v^{\prime}(x) \mathrm{d} x\right) \mathrm{d} x=f^{* *}(\alpha),
$$

where we have used Jensen's inequality. Hence

$$
J(\mathbf{u}) \geqslant \frac{E h}{2\left(1-v^{2}\right)}\left(\alpha-e_{0}\right)^{2}+f^{* *}(\alpha)=g(\alpha) \geqslant g\left(\alpha_{\min }\right),
$$

and in turn

$$
\inf _{\mathbf{u} \in W} J(\mathbf{u}) \geqslant g\left(\alpha_{\min }\right) .
$$

Next, we prove the reverse inequality. By Corollary 2.2.9 of Dacorogna [7],

$$
\begin{gathered}
f^{* *}\left(\alpha_{\min }\right)=\inf \left\{\lambda f\left(\gamma_{a}\right)+(1-\lambda) f\left(\gamma_{b}\right): \lambda \in[0,1], \gamma_{a}, \gamma_{b} \in \mathbb{R},\right. \\
\left.\lambda \gamma_{a}+(1-\lambda) \gamma_{b}=\alpha_{\min }\right\} ;
\end{gathered}
$$

thus, for any fixed $k \in \mathbb{N}$, we can find $\lambda_{k} \in[0,1]$ and $\gamma_{a, k}, \gamma_{b, k} \in \mathbb{R}$, with

$$
\alpha_{\min }=\lambda_{k} \gamma_{a, k}+\left(1-\lambda_{k}\right) \gamma_{b, k},
$$

such that

$$
f^{* *}\left(\alpha_{\min }\right) \leqslant \lambda_{k} f\left(\gamma_{a, k}\right)+\left(1-\lambda_{k}\right) f\left(\gamma_{b, k}\right) \leqslant f^{* *}\left(\alpha_{\min }\right)+\frac{1}{k} .
$$

Let $I_{a, k} \subset[0,1]$ be any measurable set with $\left|I_{a, k}\right|=\lambda_{k}$ and define $I_{b, k}:=[0,1] \backslash I_{a, k}$. Then $\left|I_{b, k}\right|=\left(1-\lambda_{k}\right)$. Let

$$
g_{k}(x)= \begin{cases}\gamma_{a, k} & x \in I_{a, k}, \\ \gamma_{b, k} & x \in I_{b, k}\end{cases}
$$

and let

$$
u_{k}(x)=\int_{0}^{x} g_{k}(t) \mathrm{d} t-\alpha_{\min } x \quad x \in[0,1] .
$$

Since $u_{k}(1)=u_{k}(0)=0$, we can extend $u_{k}$ by periodicity to all of $\mathbb{R}$. For $n \in \mathbb{N}$, let

$$
u_{n, k}(x)=\alpha_{\min } x+\frac{1}{n} u_{k}(n x) .
$$

Then, for a.e. $x \in[0,1]$,

$$
u_{n, k}^{\prime}(x)= \begin{cases}\gamma_{a, k} & n x \in I_{a, k}, \\ \gamma_{b, k} & n x \in I_{b, k}\end{cases}
$$

and, as $n \rightarrow \infty$, we may use Theorem 2.1.5 of Dacorogna [7] to conclude that

$$
u_{n, k} \stackrel{\star}{\rightarrow} \alpha_{\min } x \quad \text { in } W^{1, \infty}([0,1] ; \mathbb{R})
$$


Fix $\varepsilon>0$ and let $\psi_{\varepsilon} \in C_{0}^{\infty}([0, h] ; \mathbb{R})$ be a cut-off function, with $0 \leqslant \psi_{\varepsilon}(y) \leqslant 1$, such that $\psi_{\varepsilon}(y) \equiv 1$ on $[2 \varepsilon, h], \psi_{\varepsilon}(y) \equiv 0$ on $[0, \varepsilon]$, and

$$
\left|\psi_{\varepsilon}^{\prime}(y)\right| \leqslant C / \varepsilon \quad \text { for all } y \in[0, h] .
$$

Let

$$
\mathbf{u}_{n, \varepsilon, k}(x, y):=\left(\psi_{\varepsilon}(y) \alpha_{\min } x+\left(1-\psi_{\varepsilon}(y)\right) u_{n, k}(x)\right) \mathbf{i}-\frac{v\left(\alpha_{\min }-e_{0}\right)}{(1-v)} y \mathbf{j} .
$$

Then

$$
\left|\mathbf{u}_{n, \varepsilon, k}(x, y)-\tilde{\mathbf{u}}(x, y)\right|=\left(1-\psi_{\varepsilon}(y)\right)\left|u_{n, k}(x)-\alpha_{\min } x\right| \leqslant\left\|u_{n, k}-\alpha_{\min } x\right\|_{L^{\infty}([0,1] ; \mathbb{R})}
$$

and thus, for $k$ and $\varepsilon$ fixed,

$$
\left\|\mathbf{u}_{n, \varepsilon, k}-\tilde{\mathbf{u}}\right\|_{L^{\infty}\left(\Omega ; \mathbb{R}^{2}\right)} \rightarrow 0 \quad \text { as } n \rightarrow \infty .
$$

Moreover, by (4.5), for a.e. $(x, y) \in \Omega$,

$$
\left|\frac{\partial \mathbf{u}_{n, \varepsilon, k}}{\partial x}(x, y)-\frac{\partial \tilde{\mathbf{u}}}{\partial x}(x, y)\right|=\left(1-\psi_{\varepsilon}(y)\right)\left|u_{n, k}^{\prime}(x)-\alpha_{\min }\right| \leqslant\left(1-\psi_{\varepsilon}(y)\right)\left|\gamma_{a, k}-\gamma_{b, k}\right| .
$$

Thus, since $\psi_{\varepsilon}(y) \equiv 1$ on $[2 \varepsilon, h]$,

$$
\left\|\frac{\partial \mathbf{u}_{n, \varepsilon, k}}{\partial x}-\frac{\partial \tilde{\mathbf{u}}}{\partial x}\right\|_{L^{2}\left(\Omega ; \mathbb{R}^{2}\right)}^{2} \leqslant 2 \varepsilon\left|\gamma_{a, k}-\gamma_{b, k}\right|^{2} .
$$

Consequently, for $k$ fixed,

$$
\lim _{\varepsilon \rightarrow 0} \lim _{n \rightarrow \infty}\left\|\frac{\partial \mathbf{u}_{n, \varepsilon, k}}{\partial x}-\frac{\partial \tilde{\mathbf{u}}}{\partial x}\right\|_{L^{2}\left(\Omega ; \mathbb{R}^{2}\right)}^{2}=0 .
$$

Finally,

$$
\left|\frac{\partial \mathbf{u}_{n, \varepsilon, k}}{\partial y}(x, y)-\frac{\partial \tilde{\mathbf{u}}}{\partial y}(x, y)\right|=\left|\psi_{\varepsilon}^{\prime}(y)\right|\left|u_{n, k}(x)-\alpha_{\min } x\right| \leqslant \frac{C}{\varepsilon}\left\|u_{n, k}-\alpha_{\min } x\right\|_{L^{\infty}([0,1] ; \mathbb{R})}
$$

and thus, for $k$ and $\varepsilon$ fixed,

$$
\left\|\frac{\partial \mathbf{u}_{n, \varepsilon, k}}{\partial y}-\frac{\partial \tilde{\mathbf{u}}}{\partial y}\right\|_{L^{\infty}\left(\Omega ; \mathbb{R}^{2}\right)} \rightarrow 0 \quad \text { as } n \rightarrow \infty .
$$

In conclusion, we have shown that, for any $k$,

$$
\lim _{\varepsilon \rightarrow 0} \lim _{n \rightarrow \infty}\left\|\mathbf{u}_{n, \varepsilon, k}-\tilde{\mathbf{u}}\right\|_{W^{1,2}\left(\Omega ; \mathbb{R}^{2}\right)}=0 .
$$

Using a standard diagonalization argument, we may extract a subsequence $\mathbf{u}_{k}:=\mathbf{u}_{n_{k}, \varepsilon_{k}, k}$ which converges to $\tilde{\mathbf{u}}$ in $W^{1,2}\left(\Omega ; \mathbb{R}^{2}\right)$. Hence, as $k \rightarrow+\infty$,

$$
\lim _{k \rightarrow \infty} F\left(\mathbf{u}_{k}\right)=F(\tilde{\mathbf{u}})=\frac{E h}{2\left(1-v^{2}\right)}\left(\alpha_{\min }-e_{0}\right)^{2} .
$$


By (4.8), $\mathbf{u}_{k}(x, 0) \cdot \mathbf{i}=u_{n_{k}, k}(x) \mathbf{i}$ and thus, by (4.6),

$$
f^{* *}\left(\alpha_{\min }\right) \leqslant \int_{0}^{1} f\left(u_{n_{k}, k}^{\prime}(x)\right) \mathrm{d} x=\lambda_{k} f\left(\gamma_{a, k}\right)+\left(1-\lambda_{k}\right) f\left(\gamma_{b, k}\right) \leqslant f^{* *}\left(\alpha_{\min }\right)+\frac{1}{k} .
$$

To obtain the desired result, it suffices to let $k \rightarrow \infty$ in the previous inequality.

Proof. The existence theorem. We only prove the first assertion; the second follows directly from (3.5).

If $(\mathrm{M})$ admits a solution $\mathbf{u}$ then, by the theorem on the infimum of $J$, it must minimize the bulk energy $F(\mathbf{u})$. By the uniqueness of the minimum, it must coincide with the smooth solution $\tilde{\mathbf{u}}$ in (4.1). The trace of this function on the lower boundary $\{y=0\}$ of $\Omega$ is by construction $u(x, 0)=\alpha_{\min } x$, which must, in turn, minimize the interfacial energy functional $I(u)$. Thus

$$
f^{* *}\left(\alpha_{\min }\right)=I(u)=\int_{0}^{1} f\left(\alpha_{\min }\right) \mathrm{d} x=f\left(\alpha_{\min }\right) .
$$

Conversely, if this relation holds, the minimum exists and is given by $\tilde{\mathbf{u}}$.

Proof. The theorem on minimizing sequences. Notice first that, by (3.2) and (3.3),

$$
g_{\text {blk }}\left(\alpha_{n}\right) \leqslant F\left(\mathbf{u}_{n}\right) \quad \text { and } \quad g_{\text {int }}\left(\alpha_{n}\right) \leqslant I\left(u_{n}\right) .
$$

Thus, $g\left(\alpha_{n}\right) \leqslant J\left(\mathbf{u}_{n}\right)$. But $J\left(\mathbf{u}_{n}\right) \rightarrow g\left(\alpha_{\min }\right) \leqslant g\left(\alpha_{n}\right)$ and $g$ is strictly convex. Thus $\alpha_{n} \rightarrow \alpha_{\min }$ and we have (3.8) .

Let $\tilde{\mathbf{E}}$ be the strain associated to $\tilde{\mathbf{u}}_{\text {min }}$; then, by (4.3), we have

$$
F\left(\mathbf{u}_{n}\right)-F\left(\tilde{\mathbf{u}}_{\min }\right)=\int_{\Omega} w\left(\mathbf{E}_{n}-\tilde{\mathbf{E}}\right) \mathrm{d} x \mathrm{~d} y+\int_{\Omega} \tilde{\mathbf{T}} \cdot\left(\mathbf{E}_{n}-\tilde{\mathbf{E}}\right) \mathrm{d} x \mathrm{~d} y
$$

and applying the divergence theorem and the periodicity boundary conditions, the last integral may be written as

$$
\int_{0}^{h}\{\mathbf{i} \cdot \tilde{\mathbf{T}}(1, y) \mathbf{i}\}\left(\alpha_{n}-\alpha_{\min }\right) \mathrm{d} y
$$

which vanishes as $n \rightarrow \infty$ by (3.8) . Thus, by the positive-definiteness of the quadratic form $w(\mathbf{E})$, it follows that $\mathbf{E}_{n} \rightarrow \tilde{\mathbf{E}}$ in $L^{2}\left(\Omega, \mathbb{R}^{(2 \times 2)}\right)$, and Korn's inequality (see the appendix) yields the desired result.

\section{On the structure of minimizing sequences}

\subsection{Oscillating sequences}

The minimizing sequence (4.8) constructed in the proof of the theorem on the infimum of $J$ becomes particularly simple if we assume that there exist $\lambda \in[0,1]$ and $\gamma_{a}, \gamma_{b} \in \mathbb{R}$ such that

$$
f^{* *}\left(\alpha_{\min }\right)=\lambda f\left(\gamma_{a}\right)+(1-\lambda) f\left(\gamma_{b}\right), \quad \alpha_{\min }=\lambda \gamma_{a}+(1-\lambda) \gamma_{b}
$$

Indeed, we may then replace $g_{k}, u_{k}$, and $\mathbf{u}_{n, \varepsilon, k}$ respectively by

$$
g(x)=\left\{\begin{array}{ll}
\gamma_{a} & x \in I_{a}, \\
\gamma_{b} & x \in I_{b},
\end{array} \quad \text { with }\left|I_{a}\right|=\lambda \text { and }\left|I_{b}\right|=(1-\lambda)\right.
$$




$$
\begin{gathered}
u(x)=\int_{0}^{x} g(t) \mathrm{d} t-\alpha_{\min } x, \quad u_{n}(x)=\alpha_{\min } x+\frac{1}{n} u(n x), \\
\mathbf{u}_{n, \varepsilon}(x, y):=\left(\psi_{\varepsilon}(y) \alpha_{\min } x+\left(1-\psi_{\varepsilon}(y)\right) u_{n}(x)\right) \mathbf{i}-\frac{v\left(\alpha_{\min }-e_{0}\right)}{(1-v)} y \mathbf{j} .
\end{gathered}
$$

Note that, when

$$
f^{* *}\left(\alpha_{\min }\right)<f\left(\alpha_{\min }\right)
$$

each function $u_{n}(x)$ is a minimizer of the problem

$$
\inf _{\hat{W}_{\alpha}} I(u)=\frac{\gamma_{b}-\alpha_{\min }}{\gamma_{b}-\gamma_{a}} f\left(\gamma_{a}\right)+\frac{\alpha_{\min }-\gamma_{a}}{\gamma_{b}-\gamma_{a}} f\left(\gamma_{b}\right),
$$

since

$$
u_{n}^{\prime}(x)= \begin{cases}\gamma_{a} & n x \in I_{a}, \\ \gamma_{b} & n x \in I_{b}\end{cases}
$$

but the weak limit of the sequence, $\alpha_{\min } x$, is not.

This is why the minimization problem $(M)$ has no classical solution, and the infimum of the interfacial energy is reached only in a generalized sense, a sense well described by Young measures.

This approach has been used successfully for minimization problems in phase transitions (see e.g. the work of Ball \& James [2]).

In our case, we can show that the sequence $\gamma_{n}$ generates the gradient $W^{1, \infty}$-Young measure (in the sense of Kinderlehrer \& Pedregal [16])

$$
v=\mathrm{d} x \otimes\left(\lambda \delta_{\gamma_{a}}+(1-\lambda) \delta_{\gamma_{b}}\right)
$$

where $\delta_{\gamma_{a}}$ and $\delta_{\gamma_{b}}$ are the Dirac measures on $\mathbb{R}$ concentrated respectively on $\gamma_{a}$ and $\gamma_{b}$.

Indeed the sequence $u_{n}$ is weakly* convergent in $W^{1, \infty}([0,1] ; \mathbb{R})$. Moreover, for any $\varphi \in$ $C_{0}([0,1] \times \mathbb{R} ; \mathbb{R})$,

$$
\begin{array}{r}
\int_{0}^{1} \varphi\left(x, \gamma_{n}(x)\right) \mathrm{d} x=\int_{0}^{1} \chi_{a}(n x) \varphi\left(x, \gamma_{a}\right) \mathrm{d} x+\int_{0}^{1} \chi_{b}(n x) \varphi\left(x, \gamma_{b}\right) \mathrm{d} x \\
\rightarrow \int_{0}^{1} \lambda \varphi\left(x, \gamma_{a}\right) \mathrm{d} x+\int_{0}^{1}(1-\lambda) \varphi\left(x, \gamma_{b}\right) \mathrm{d} x=\int_{[0,1] \times \mathbb{R}} \varphi \mathrm{d} v
\end{array}
$$

as $n \rightarrow \infty$, where $\chi_{a}$ and $\chi_{b}$ are the characteristic functions of the sets $I_{a}$ and $I_{b}$, respectively, and we have used Theorem 2.1.5 of Dacorogna [7] to conclude that

$$
\begin{array}{ll}
\chi_{a}(n x) \stackrel{\star}{\rightarrow} \int_{0}^{1} \chi_{a}(x) \mathrm{d} x=\lambda & \text { in } L^{\infty}([0,1] ; \mathbb{R}), \\
\chi_{b}(n x) \stackrel{\star}{\rightarrow} \int_{0}^{1} \chi_{b}(x) \mathrm{d} x=(1-\lambda) & \text { in } L^{\infty}([0,1] ; \mathbb{R}) .
\end{array}
$$




\subsection{Uniqueness of the Young measure}

In this section we prove that under some general hypotheses on the function $f$ all minimizing sequences generate the same Young measure at the interface. The approach is classical: see e.g. the work of Ball \& James [3]).

We assume that

$$
\lim _{\gamma \rightarrow \infty} \frac{f(\gamma)}{\gamma}=\infty
$$

Let $\left\{\mathbf{u}_{n}\right\}$ be a minimizing sequence for the problem $(M)$ with $\alpha_{n}$ the average incoherency strain associated with $\mathbf{u}_{n}$. We have already proved that

$$
\begin{aligned}
& \alpha_{n} \rightarrow \alpha_{\min }, \quad \int_{0}^{1} f\left(\gamma_{n}\right) \mathrm{d} x \rightarrow f^{* *}\left(\alpha_{\min }\right), \\
& \mathbf{u}_{n} \rightarrow \tilde{\mathbf{u}}_{\min } \quad \text { in } \quad W^{1,2}\left(\Omega, \mathbb{R}^{2}\right),
\end{aligned}
$$

where $\tilde{\mathbf{u}}_{\text {min }}$ is defined in (3.9). By the growth condition (5.4) and (5.5) $)_{2}$, we have, in particular, that

$$
\int_{0}^{1}\left|\gamma_{n}\right| \mathrm{d} x \leqslant M
$$

for all $n \in \mathbb{N}$. In turn

$$
\left|u_{n}(x)\right| \leqslant \int_{0}^{1}\left|\gamma_{n}\right| \mathrm{d} x \leqslant M .
$$

Hence, again by (5.4), (5.5) 2 , and the Dunford-Pettis criterion, there exists a subsequence $u_{n_{k}}$ which converges weakly to some function $v$ in the space $W^{1,1}([0,1], \mathbb{R})$. On the other hand, since by the continuity of the trace operator $u_{n}$ converges to $\alpha_{\min } x$ in $L^{2}([0,1], \mathbb{R})$, then, necessarily, $v(x)=$ $\alpha_{\min } x$ and thus the entire sequence $u_{n}$ converges weakly to $\alpha_{\min } x$ in $W^{1,1}([0,1], \mathbb{R})$. There are now two cases.

If $f^{* *}\left(\alpha_{\min }\right)=f\left(\alpha_{\min }\right)$, then $\tilde{\mathbf{u}}_{\min }$ is a classical solution of the problem $(M)$. Thus we may focus on the complementary situation

$$
f^{* *}\left(\alpha_{\min }\right)<f\left(\alpha_{\min }\right)
$$

Let

$$
A_{\min }:=\left\{\gamma \in \mathbb{R}: f^{* *}(\gamma)=f^{* *}\left(\alpha_{\min }\right)+\left(f^{* *}\right)_{+}^{\prime}\left(\alpha_{\min }\right)\left(\gamma-\alpha_{\min }\right)\right\} .
$$

The set $A_{\min }$ is clearly closed, convex, and nonempty, since $\alpha_{\min } \in A_{\min }$. Moreover it is also bounded, since by (5.4)

$$
\lim _{\gamma \rightarrow \infty} \frac{f^{* *}(\gamma)}{\gamma}=\infty
$$

Thus $A_{\min }=\left[\gamma_{a}, \gamma_{b}\right]$, where $0 \leqslant \gamma_{a} \leqslant \alpha_{\min } \leqslant \gamma_{b}$. If $\gamma_{a}<\gamma_{b}$, then

$$
f\left(\gamma_{a}\right)=f^{* *}\left(\gamma_{a}\right) \quad \text { and } \quad f\left(\gamma_{b}\right)=f^{* *}\left(\gamma_{b}\right) .
$$

Indeed, suppose, for example, that $f\left(\gamma_{b}\right)>f^{* *}\left(\gamma_{b}\right)$. By continuity we can find $\rho>0$ and $0<$ $\varepsilon_{0}<\gamma_{b}-\gamma_{a}$ such that

$$
f(\gamma)>f^{* *}(\gamma)+\rho \quad \text { for all } \gamma \in\left[\gamma_{b}-\varepsilon_{0}, \gamma_{b}+\varepsilon_{0}\right] .
$$


Consider the function

$$
f_{1}(\gamma)=f^{* *}\left(\gamma_{b}-\varepsilon\right)+\frac{f^{* *}\left(\gamma_{b}+\varepsilon\right)-f^{* *}\left(\gamma_{b}-\varepsilon\right)}{2 \varepsilon}\left(\gamma-\left(\gamma_{b}-\varepsilon\right)\right),
$$

where we have chosen $0<\varepsilon \leqslant \varepsilon_{0}$ so small that

$$
f^{* *}(\gamma)<f_{1}(\gamma) \leqslant f(\gamma)-\frac{1}{2} \rho \quad \text { for all } \gamma \in\left(\gamma_{b}-\varepsilon, \gamma_{b}+\varepsilon\right)
$$

The function

$$
f_{2}(\gamma):=\sup \left\{f_{1}(\gamma), f^{* *}(\gamma)\right\}= \begin{cases}f_{1}(\gamma) & \text { in }\left[\gamma_{b}-\varepsilon, \gamma_{b}+\varepsilon\right] \\ f^{* *}(\gamma) & \text { otherwise }\end{cases}
$$

is convex and such that $f^{* *}(\gamma) \leqslant f_{2}(\gamma) \leqslant f(\gamma)$ for all $\gamma \in \mathbb{R}$, with the inequalities strict in $\left(\gamma_{b}-\varepsilon, \gamma_{b}+\varepsilon\right)$. This is a contradiction. Thus (5.7) holds. In this case, by (5.6), we have $\gamma_{a}<$ $\alpha_{\min }<\gamma_{b}$ so that $f^{* *}$ is actually differentiable at $\alpha_{\min }$.

When $\gamma_{a}<\gamma_{b}$, we make the essential hypothesis that

$$
f^{* *}(\gamma)<f(\gamma) \quad \text { in }\left(\gamma_{a}, \gamma_{b}\right)
$$

We claim that

$$
\operatorname{dist}\left(\gamma_{n},\left\{\gamma_{a}\right\} \cup\left\{\gamma_{b}\right\}\right) \rightarrow 0 \quad \text { in measure. }
$$

Indeed, fix $\varepsilon>0$ (with $0<\varepsilon<\gamma_{b}-\gamma_{a}$ if $\gamma_{a}<\gamma_{b}$ ). Then, by the equintegrability of the sequence $\gamma_{n}$ there exists $M=M(\varepsilon)>\gamma_{b}+1$ such that

$$
\left|\left\{x \in[0,1]: \quad\left|\gamma_{n}(x)\right| \geqslant M\right\}\right| \leqslant \varepsilon / 3 .
$$

If $\gamma_{a}<\gamma_{b}$ let

$$
C_{\varepsilon}=\min \left\{f(\gamma)-f^{* *}(\gamma): \quad \gamma \in\left[\gamma_{a}+\varepsilon, \gamma_{b}-\varepsilon\right]\right\} .
$$

Then $C_{\varepsilon}>0$ by (5.8). Moreover, since $u_{n}$ converges weakly to $\alpha_{\min } x$ in $W^{1,1}([0,1], \mathbb{R})$, by classical lower semicontinuity results and Jensen's inequality we have

$$
\begin{aligned}
f^{* *}\left(\alpha_{\min }\right) & =\lim _{n \rightarrow \infty} \int_{0}^{1} f\left(\gamma_{n}\right) \mathrm{d} x \\
& \geqslant \liminf _{n \rightarrow \infty} \int_{0}^{1} f^{* *}\left(\gamma_{n}\right) \mathrm{d} x \geqslant \int_{0}^{1} f^{* *}\left(\alpha_{\min }\right) \mathrm{d} x=f^{* *}\left(\alpha_{\min }\right) .
\end{aligned}
$$

Consequently

$$
0=\lim _{n \rightarrow \infty} \int_{0}^{1}\left[f\left(\gamma_{n}\right)-f^{* *}\left(\gamma_{n}\right)\right] \mathrm{d} x \geqslant C_{\varepsilon} \lim _{n \rightarrow \infty}\left|\left\{x \in[0,1]: \quad \gamma_{n}(x) \in\left[\gamma_{a}+\varepsilon, \gamma_{b}-\varepsilon\right]\right\}\right| .
$$

Let

$$
\begin{aligned}
D_{\varepsilon}=\min \left\{f^{* *}(\gamma)-f^{* *}\left(\alpha_{\min }\right)-(\right. & \left(f^{* *}\right)^{\prime}\left(\alpha_{\min }\right)\left(\gamma-\alpha_{\min }\right) \\
& \left.: \gamma \in\left[\gamma_{b}+\varepsilon, M\right] \cup\left[-M, \gamma_{a}-\varepsilon\right]\right\}
\end{aligned}
$$


Then $D_{\varepsilon}>0$ by the definition of the set $A_{\min }$. Moreover, since $\alpha_{n} \rightarrow \alpha_{\min }$,

$$
\begin{aligned}
f^{* *}\left(\alpha_{\min }\right)=\lim _{n \rightarrow \infty} & \int_{0}^{1} f^{* *}\left(\gamma_{n}\right) \mathrm{d} x \\
& \geqslant \liminf _{n \rightarrow \infty} \int_{0}^{1}\left[f^{* *}\left(\alpha_{\min }\right)+\left(f^{* *}\right)^{\prime}\left(\alpha_{\min }\right)\left(\gamma_{n}-\alpha_{\min }\right)\right] \mathrm{d} x=f^{* *}\left(\alpha_{\min }\right) .
\end{aligned}
$$

Thus

$$
\begin{aligned}
0=\lim _{n \rightarrow \infty} & \int_{0}^{1}\left[f^{* *}\left(\gamma_{n}\right)-f^{* *}\left(\alpha_{\min }\right)-\left(f^{* *}\right)^{\prime}\left(\alpha_{\min }\right)\left(\gamma_{n}-\alpha_{\min }\right)\right] \mathrm{d} x \\
& \geqslant D_{\varepsilon} \lim _{n \rightarrow \infty}\left|\left\{x \in[0,1]: \quad \gamma_{n}(x) \in\left[\gamma_{b}+\varepsilon, M\right] \cup\left[-M, \gamma_{a}-\varepsilon\right]\right\}\right| .
\end{aligned}
$$

In conclusion we have shown that, for $n$ sufficiently large,

$$
\left|\left\{x \in[0,1]: \quad \operatorname{dist}\left(\gamma_{n},\left\{\gamma_{a}\right\} \cup\left\{\gamma_{b}\right\}\right) \geqslant \varepsilon\right\}\right| \leqslant \varepsilon,
$$

that is (5.9).

Since $\gamma_{n}$ converges weakly to $\alpha_{\min }$ in $L^{1}([0,1], \mathbb{R})$, we can apply the fundamental theorem on Young measure (see e.g. [14]) to obtain the existence of a weak* measurable map $v:[0,1] \rightarrow$ $\mathcal{M}(\mathbb{R})$ such that the following hold:

(i) $v_{x} \geqslant 0,\left\|v_{x}\right\|_{\mathcal{M}(\mathbb{R})}=\int_{\mathbb{R}} \mathrm{d} v_{x}=1$ for a.e. $x \in[0,1]$;

(ii) supp $v_{x} \subset\left\{\gamma_{a}\right\} \cup\left\{\gamma_{b}\right\}$ for a.e. $x \in[0,1]$.

Thus for a.e. $x \in[0,1]$

$$
v_{x}=\lambda(x) \delta_{\gamma_{a}}+(1-\lambda(x)) \delta_{\gamma_{b}}, \quad \text { where } \quad 0 \leqslant \lambda(x) \leqslant 1 .
$$

On the other hand, since

$$
\alpha_{\min } \equiv\left\langle v_{x}, \mathrm{id}\right\rangle=\int_{\mathbb{R}} y \mathrm{~d} v_{x}(y)=\lambda(x) \gamma_{a}+(1-\lambda(x)) \gamma_{b}
$$

it follows that $\lambda(x) \equiv \lambda$. Hence

$$
v_{x}=\lambda \delta_{\gamma_{a}}+(1-\lambda) \delta_{\gamma_{b}}
$$

and the proof is complete.

\subsection{Concentrating sequences}

Condition (5.1) may not be satisfied in the important case in which $f$ is strictly concave. We have

$$
f^{* *}(\gamma)=m \gamma, \quad \text { with } \quad m=\lim _{\gamma \rightarrow+\infty} \frac{f(\gamma)}{\gamma} .
$$

This may be proved using the inequalities $f^{* *}(\lambda \gamma) \leqslant \lambda f^{* *}(\gamma) \leqslant \lambda f(\gamma) \leqslant f(\lambda \gamma)$ : taking $\lambda=$ $\bar{\gamma} / \gamma<1$ we obtain that

$$
\frac{f^{* *}(\bar{\gamma})}{\bar{\gamma}} \leqslant \frac{f(\gamma)}{\gamma} \leqslant \frac{f(\bar{\gamma})}{\bar{\gamma}}
$$


and, taking the limit as $\gamma \rightarrow+\infty$, we have the above representation for the convex envelope of $f$. In this case, $\alpha_{\min }$ can be computed explicitly. Indeed

$$
\alpha_{\min }=\max \left\{0, e_{0}-\frac{m\left(1-v^{2}\right)}{E h}\right\},
$$

so that (5.1) fails when

$$
h>\frac{m\left(1-v^{2}\right)}{E e_{0}},
$$

because $\alpha_{\min }>0$.

To construct the minimizing sequence (4.8) in the proof of the theorem on the infimum of $J$, note that, if $\gamma_{a, k} \leqslant \gamma_{b, k}$, then

$$
\lim _{k \rightarrow+\infty} \gamma_{a, k}=0, \quad \lim _{k \rightarrow+\infty} \gamma_{b, k}=+\infty .
$$

To see this, observe that by the strict concavity of $f, f(\gamma) / \gamma$ is strictly decreasing, so that

$$
\begin{aligned}
& m \alpha_{\min } \leqslant \frac{f\left(\gamma_{b, k}\right)}{\gamma_{b, k}} \alpha_{\min } \leqslant \lambda_{k} f\left(\gamma_{a, k}\right)+\left(1-\lambda_{k}\right) f\left(\gamma_{b, k}\right), \\
& m \alpha_{\min } \leqslant \lambda_{k}\left(f\left(\gamma_{a, k}\right)-m \gamma_{b, k}\right)+m \alpha_{\min } \leqslant \lambda_{k} f\left(\gamma_{a, k}\right)+\left(1-\lambda_{k}\right) f\left(\gamma_{b, k}\right) .
\end{aligned}
$$

Hence, to attain the infimimum, a minimizing sequence must be such that

$$
\lim _{k \rightarrow+\infty} \frac{f\left(\gamma_{b, k}\right)}{\gamma_{b, k}}=m
$$

and (5.10) $)_{2}$ follows. Now, $\gamma_{a, k}$ is bounded; otherwise, by the growth properties of $f$, the expression $\lambda_{k} f\left(\gamma_{a, k}\right)+\left(1-\lambda_{k}\right) f\left(\gamma_{b, k}\right)$ would be unbounded; and this shows that

$$
\lambda_{k}=\frac{\gamma_{b, k}-\alpha_{\min }}{\gamma_{b, k}-\gamma_{a, k}} \rightarrow 1,
$$

which implies

$$
\lim _{k \rightarrow+\infty}\left(f\left(\gamma_{a, k}\right)-m \gamma_{a, k}\right)=0,
$$

which proves $(5.10)_{1}$.

For instance, we may take, as a minimizing sequence,

$$
g_{k}(x)= \begin{cases}\alpha_{\min } k & x \in[0,1 / k] \\ 0 & x \in[1 / k, 1]\end{cases}
$$

so that, for a.e. $x \in[0,1]$,

$$
u_{k}^{\prime}(x)=u_{n_{k}, k}^{\prime}(x)= \begin{cases}\alpha_{\min } k & n_{k} x \in[0,1 / k], \\ 0 & n_{k} x \in[1 / k, 1] .\end{cases}
$$

Contrary to the previous subsection, Young measures cannot be used in the present context to describe the structure of the minimizing sequence, since they cannot describe concentrational 
effects. Instead, we use here the concepts of varifolds or indicator measures (see e.g. the work of Fonseca [12] and of Fonseca, Müller \& Pedregal [13]). We claim that the sequence $\gamma_{n}$ defined in (5.11) generates the $W^{1,1}$-varifold

$$
\Lambda=\mathrm{d} x \otimes \alpha_{\min } \delta_{1},
$$

where $\delta_{1}$ is the Dirac measure on the unit sphere $S=\{-1,1\}$ concentrated on 1 . Indeed the sequence $u_{k}$ is bounded in $W^{1,1}([0,1] ; \mathbb{R})$. Moreover, for any $\varphi \in C_{0}([0,1] \times \mathbb{R} ; \mathbb{R})$, by the mean-value theorem,

$$
\begin{gathered}
\int_{0}^{1} \varphi\left(x, \frac{\gamma_{k}(x)}{\left|\gamma_{k}(x)\right|}\right)\left|\gamma_{k}(x)\right| \mathrm{d} x=\alpha_{\min } k \int_{0}^{1} \chi_{k}\left(n_{k} x\right) \varphi(x, 1) \mathrm{d} x \\
=\alpha_{\min } \sum_{h=0}^{n_{k}-1} k \int_{h / n_{k}}^{(h+1 / k) / n_{k}} \varphi(x, 1) \mathrm{d} x=\alpha_{\min } \sum_{h=0}^{n_{k}-1} \frac{1}{n_{k}} \varphi\left(x_{h, k}, 1\right) \\
\rightarrow \alpha_{\min } \int_{0}^{1} \varphi(x, 1) \mathrm{d} x=\int_{[0,1] \times S} \varphi \mathrm{d} \Lambda \quad \text { as } k \rightarrow \infty,
\end{gathered}
$$

where $\chi_{k}$ is the characteristic functions of the set $[0,1 / k]$, and

$$
x_{h, k} \in\left[\frac{h}{n_{k}}, \frac{h}{n_{k}}+\frac{1}{k n_{k}}\right] \subset\left[\frac{h}{n_{k}}, \frac{h+1}{n_{k}}\right] .
$$

The claim is thus proved.

It is clear that one can also take $\varphi(x, \gamma)=\theta(x) \phi(\gamma)$, where $\theta \in C_{0}([0,1] ; \mathbb{R})$ and $\phi$ is positively homogeneous of degree one. Thus we can use the varifold to express the infimum of the interfacial energy in a suggestive form. Let $\theta \in C_{0}([0,1] ; \mathbb{R})$; then

$$
\int_{0}^{1} \theta(x) f\left(\gamma_{k}\right) \mathrm{d} x=\int_{0}^{1} \theta(x)\left(f\left(\gamma_{k}\right)-m \gamma_{k}\right) \mathrm{d} x+\int_{0}^{1} \theta(x) m \gamma_{k} \mathrm{~d} x .
$$

The first integral on the right-hand side goes to zero as $k \rightarrow \infty$, since

$$
\left|\int_{0}^{1} \theta(x)\left(f\left(\gamma_{k}\right)-m \gamma_{k}\right) \mathrm{d} x\right| \leqslant \alpha_{\min }\left(\frac{f\left(\alpha_{\min } k\right)}{\alpha_{\min } k}-m\right)\|\theta\|_{\infty} .
$$

Now, choosing $\varphi(x, \gamma)=\theta(x) m \gamma$, we obtain

$$
\int_{0}^{1} \theta(x) f\left(\gamma_{k}\right) \mathrm{d} x \rightarrow m \alpha_{\min } \int_{0}^{1} \theta \mathrm{d} x=\int_{[0,1] \times S} \theta f^{* *} \mathrm{~d} \Lambda .
$$

\section{Results for specific forms of the interfacial energy}

We now turn to the analysis of various forms of the interfacial energy density $f$. We will focus on the problem of the dependence of $\alpha_{\min }$ on $h$, where, we recall, $\alpha_{\min }$ is the average incoherency strain corresponding to the infimum of $J$, which is defined in (3.4). As we have shown in the existence theorem, the non-smoothness of $f^{* *}$ at zero implies the existence of a critical value for $h$ below which the interface is coherent. Analogously, non-smoothness at other points implies that there are intervals of $h$ within which the interface remains smoothly incoherent with a fixed incoherency strain. Finally, when $f^{* *}$ is smooth at $\alpha_{\min }$, the interface is either finely or smoothly incoherent according to the convexity at $\alpha_{\min }$. 


\section{1 f convex and smooth (Fig. 1)}

Since, in this case, $f(\gamma)=f^{* *}(\gamma)$, the solution exists in $W$ and is given by $\tilde{\mathbf{u}}_{\min }$ in (3.9). The trace of $\tilde{\mathbf{u}}_{\min }$ on the lower boundary of $\Omega$ is $u(x)=\alpha_{\min } x$, with $\alpha_{\min }$ the solution of (3.4).

Thus the interface is always smoothly incoherent, since the film is uniformly strained with respect to the substrate, and no fine structure appears.

The average strain at the interface $\alpha_{\min }$, which measures incoherency, is related to the variation of the thickness of the layer by the relation

$$
h=\frac{1-v^{2}}{E} \frac{f^{\prime}\left(\alpha_{\min }\right)}{e_{0}-\alpha_{\min }},
$$

which shows that, when $h \rightarrow 0$, then $\alpha_{\min } \rightarrow 0$, and when $h \rightarrow+\infty$, then $\alpha_{\min } \rightarrow e_{0}$. Note that, by (3.7), the critical thickness $h_{c}$ vanishes, and the interface can never be coherent.

\section{$6.2 f$ convex but non-smooth at $\gamma=0$ (Fig. 2)}

Again $f(\gamma)=f^{* *}(\gamma)$, so the solution exists in $W$, and no fine structure develops at the interface, which can thus only be either coherent or smoothly incoherent.

Since $f$ is not differentiable at $\gamma=0$, there exists a critical thickness for the transition to incoherency, which is given by (cf. (3.7))

$$
h_{c}:=\frac{1-v^{2}}{E} \frac{f_{+}^{\prime}(0)}{e_{0}} .
$$

Thus for $h \leqslant h_{c}$ the interface is coherent, while for $h>h_{c}$ the interface is uniformly strained with respect to the substrate, and relation (6.1) still holds.

\subsection{Leo-Hu energy (Fig. 3)}

In this case, there is a value $\gamma_{1}$ of the incoherency strain such that $f$ is concave for $|\gamma|<\gamma_{1}$, and convex otherwise. Moreover, $f$ is non-smooth at $\gamma=0$, as is the convex envelope of $f$, which is

$$
f^{* *}(\gamma)= \begin{cases}m|\gamma| & |\gamma| \leqslant \gamma_{1}, \\ f(\gamma) & |\gamma|>\gamma_{1},\end{cases}
$$

with $m=\frac{f\left(\gamma_{1}\right)}{\gamma_{1}}$. Thus there is a critical thickness for coherency given by

$$
h_{c}:=\frac{1-v^{2}}{E} \frac{m}{e_{0}}
$$

such that for $h \leqslant h_{c}$ the interface is coherent.

Two cases are now possible. Assume first that the mismatch strain is small, in particular that

$$
e_{0}<\gamma_{1}
$$

so that, since $\alpha_{\min }<e_{0}$, then $f^{* *}\left(\alpha_{\min }\right)<f\left(\alpha_{\min }\right)$, and thus for $h>h_{c}$ the interface is finely incoherent. Moreover, writing $\alpha_{\min }=\lambda \gamma_{1}$, then $f^{* *}\left(\alpha_{\min }\right)=m \lambda \gamma_{1}=\lambda f\left(\gamma_{1}\right)$, so that (5.1) holds, and the minimizing sequence is constructed as in (5.2) taking $\gamma_{a}=0$ and $\gamma_{b}=\gamma_{1}$. 
Thus, when $h$ is above the critical thickness and coherency is lost by not being energetically favoured, the energy is decreased towards its infimum by interfaces composed of finer and finer mixtures of coherent and incoherent patches, with total length fractions $1-\alpha_{\min } / \gamma_{1}$ and $\alpha_{\min } / \gamma_{1}$ respectively. This sequence determines a Young measure, which characterizes its oscillation properties and thus ultimately the fine structure of the interface.

On the other hand, when

$$
e_{0}>\gamma_{1}
$$

we may define a second critical thickness

$$
h_{c}^{\prime}:=\frac{1-v^{2}}{E} \frac{m}{e_{0}-\gamma_{1}},
$$

such that, for $h>h_{c}^{\prime}$, then $\gamma_{1}<\alpha_{\min }<e_{0}$, and the interface becomes smoothly incoherent, since $f^{* *}\left(\alpha_{\min }\right)=f\left(\alpha_{\min }\right)$ and $(\mathrm{M})$ has a regular solution in $W$. Below $h_{c}^{\prime}$ the interface is finely incoherent as before.

In other words, when the mismatch strain is large, the interface loses coherency at the first critical thickness by nucleating finer and finer incoherent patches, but when the second critical thickness is reached, then this fine structure is lost and the layer becomes uniformly strained with respect to the substrate.

\section{4 f nonconvex and nonsmooth (Fig. 4)}

Here there are values $0<\gamma_{1}<\gamma_{2}<\ldots$ of the incoherency strain such that $f^{* *}(\gamma)=f(\gamma)$ at and only at $\gamma= \pm \gamma_{i} . f^{* *}(\gamma)$ is therefore piecewise linear with slope changes when $\gamma= \pm \gamma_{i}$ :

$$
f^{* *}(\gamma)= \begin{cases}m_{1}|\gamma| & |\gamma| \leqslant \gamma_{1}, \\ m_{2}|\gamma|+\text { const. } & \gamma_{1} \leqslant|\gamma| \leqslant \gamma_{2}, \\ \cdots & \end{cases}
$$

with $m_{1}<m_{2}$. To fix ideas, assume that the mismatch strain is such that $e_{0} \in\left(\gamma_{1}, \gamma_{2}\right)$ : then (3.7) shows that, when the thickness is below the first critical value given by

$$
h<h_{c}:=\frac{1-v^{2}}{E} \frac{m_{1}}{e_{0}},
$$

the interface remains coherent.

To proceed further, define two more critical values for $h$ by

$$
h_{c}^{\prime}:=\frac{1-v^{2}}{E} \frac{m_{1}}{e_{0}-\gamma_{1}}, \quad h_{c}^{\prime \prime}:=\frac{1-v^{2}}{E} \frac{m_{2}}{e_{0}-\gamma_{1}},
$$

which have the following properties; for $h$ such that

$$
h_{c}<h<h_{c}^{\prime},
$$

the interface is finely incoherent: (5.1) holds, and the energy is minimized by sequences as in (5.2), oscillating between $\gamma_{a}=0$ and $\gamma_{b}=\gamma_{1}$ (with total volume fraction $\alpha_{\min } / \gamma_{1}$ ), and which represent 
mixtures of coherent and incoherent patches. The fine structure of the interface is summarized by a Young measure, as in the preceding section.

When the thickness reaches the second critical value, i.e. for

$$
h_{c}^{\prime}<h<h_{c}^{\prime \prime}
$$

the interface structure changes drastically and it becomes smoothly incoherent. Indeed, for all values of the thickness satisfying the above inequality, the relative incoherency strain remains constant and fixed at the value $\gamma \equiv \gamma_{1}$.

The layer remains 'glued' to the substrate with fixed incoherency strain until the third critical threshold is reached. Beyond this, i.e. for a thickness such that

$$
h>h_{c}^{\prime \prime}
$$

oscillations appear again, and the interface becomes finely incoherent. In particular, since (5.1) holds, the minimizing sequences correspond to fine mixtures of different incoherent patches, corresponding to incoherency strains $\gamma_{a}=\gamma_{1}$ and $\gamma_{b}=\gamma_{2}$, and the fine structure of the interface is again determined by a Young measure.

This behaviour is reminiscent of 'coincidence boundaries', which are special incoherent interfaces between crystals with a large difference in lattice parameters. In these cases, a stable configuration is attained when the incoherency strain assumes a given value, corresponding to the ratio of the lattice parameters (say $\gamma_{1}=p / q>1$, with $p$ and $q$ integers). As the thickness changes, the interface tends to stick to this particular structure, and this is accounted for by the critical thickness interval $\left[h_{c}^{\prime}, h_{c}^{\prime \prime}\right]$. When the threshold $h_{c}^{\prime \prime}$ is reached, the interface becomes a fine mixture of regions at which the relative strain oscillates between $\gamma_{1}$ and a neighbouring minimum for the energy, say $\gamma_{2}=(p-1) /(q-1)$.

\section{5 $f$ strictly concave and non-smooth at $\gamma=0$ (Fig. 5)}

We have seen that, in this case,

$$
f^{* *}(\gamma)=m \gamma, \quad \text { with } \quad m=\lim _{\gamma \rightarrow+\infty} \frac{f(\gamma)}{\gamma},
$$

so that

$$
\alpha_{\min }=\max \left\{0, e_{0}-\frac{m\left(1-v^{2}\right)}{E h}\right\}
$$

Thus, if

$$
0<h<h_{c}=\frac{m\left(1-v^{2}\right)}{E e_{0}},
$$

the interface is coherent, and we have again a threshold effect. Above the critical thickness $h_{c}$, $\alpha_{\min }>0$ and the interface is incoherent, but since $f^{* *}<f$, the minimum of the energy is not reached by a smooth function in $W$ : the interface is finely incoherent.

Moreover, (5.1) does not hold, and the minimizing sequences are as in (5.11), so that the incoherency strain $\gamma$ tends to concentrate and become unbounded on sets of measure approaching zero. This should model the formation and fine dispersion of dislocations along the interface, these being naively represented by jumps in the tangential displacement. 
Since no regular fine structure is actually present, the fine properties of the interface (and of the minimizing sequences) are best summarized by the varifold computed in the preceding section. The varifold is constant along the interface, which shows that the 'defects' are uniformly distributed.

Notice finally that, for this family of interfacial energies, the threshold effect for the transition between coherency and incoherency, and thus the ability of the interface to support shear stresses, is essentially determined by the growth rate of $f$ at infinity, as described by $m$. This may be explained by the fact that, as noticed above, the minimizing sequences are characterized by concentrations of the incoherency strain on smaller and smaller sets at which it becomes unbounded.

\subsection{The convex envelope of $f$ is identically zero}

This situation occurs if and only if $\liminf _{\gamma \rightarrow \infty} \frac{f(\gamma)}{\gamma}=0$, so that (5.1) does not hold.

In this case $f^{* *} \equiv 0$, and

$$
\alpha_{\min }=e_{0}>0 \text {. }
$$

The interface is always finely incoherent, since the critical thickness vanishes, and relaxes completely to the bulk equilibrium strain, so that the average incoherency strain coincides with the mismatch strain.

If $f$ is concave, the infimum of the energy is not attained, and the structure of the minimizing sequences is as above, with incoherency strain concentrating on sets of measure zero. The fine properties of the interface are described again by a varifold as in Section 6.5.

To construct the minimizing sequences (4.8) in the proof of the Theorem on the Infimum of $J$, let $t_{k} \nearrow \infty$ be such that

$$
\lim _{k \rightarrow \infty} \frac{f\left(t_{k}\right)}{t_{k}}=0
$$

Then we may choose, for example,

$$
g_{k}(x)= \begin{cases}e_{0} t_{k} & x \in\left[0,1 / t_{k}\right] \\ 0 & x \in\left[1 / t_{k}, 1\right]\end{cases}
$$

so that

$$
u_{k}^{\prime}(x)=u_{n_{k}, k}^{\prime}(x)= \begin{cases}e_{0} t_{k} & n_{k} x \in\left[0,1 / t_{k}\right] \\ 0 & n_{k} x \in\left[1 / t_{k}, 1\right]\end{cases}
$$

\section{Acknowledgements}

The work of Gurtin was supported by the U.S. Department of Energy and the U.S. National Science Foundation. Cermelli and Leoni have been partially supported by the M.U.R.S.T. Project 'Modelli Matematici per la Scienza dei Materiali' and the C.N.R. strategic project 'Modelli e Metodi per la Matematica e l'Ingegneria' respectively. 


\section{REFERENCES}

1. AdAms, R. Sobolev spaces. Academic Press, New York (1975).

2. BALl, J. M. \& JameS, R. D. Fine phase mixtures as minimizers of energy. Arch. Rat. Mech. Anal. 100, (1987) 13-52.

3. BALl, J. M. \& JAmeS, R. D. Proposed experimental test of a theory of fine microstructure and the two-well problem. Phil. Trans. R. Soc. A 338, (1992) 389-450.

4. CAhn, J. W. \& LARChÉ, F. C. Surface stress and the chemical equilibrium of small crystals II. Solid particles embedded in a solid matrix. Acta Metallurgica 30, (1982) 51-56.

5. Cermelli, P. \& Gurtin, M. E. The dynamics of solid-solid phase transitions. 2 Incoherent interfaces. Arch. Rat. Mech. Anal. 127, (1994) 41-99.

6. Cermelli, P. \& Gurtin, M. E. On the kinematics of coherent and incoherent phase transitions. Acta Metallurgica et Materia 42(10), (1994) 3349-3359.

7. Dacorogna, B. Direct methods in the calculus of variations. Springer, Berlin (1989).

8. DAUTRAY, R. \& LIONS, J. L. Mathematical analysis and numerical methods for science and technology, vol. 2. Springer, Berlin (1988).

9. Du Plessis, J. C. \& VAN Der Merwe, J. H. On pointed minima in the interfacial energy of bicrystal systems. Phil. Mag. ser. 8 11, (1965) 43-51,

10. Fletcher, N. H. Structure and energy of crystal interfaces II. A simple explicit calculation. Phil. Mag. ser. 8 16, (1967) 159-164.

11. Fletcher, N. H. \& AdAmson, P. L. Structure and energy of crystal interfaces I. Formal development, Phil. Mag. ser. 8 14, (1966) 99-110.

12. Fonseca, I. Lower semicontinuity of surface energies. Proc. Roy. Soc. A 120, (1992) 99-115.

13. Fonseca, I., MÜller, S., \& Pedregal, P. Analysis of concentration and oscillation effects generated by gradients. SIAM J. Math. Anal. 29, (1997) 736-756.

14. FonSECA, I. \& MÜLLER, S. A-quasiconvexity, lower semicontinuity and Young measures. Preprint n.181998, Max-Planck-Institut, Leipzig.

15. GRISVARD, P. Elliptic problems in nonsmooth domains. Pitman, Boston (1985).

16. Kinderlehrer, D. \& Pedregal, P. Gradient Young measures generated by sequences in Sobolev spaces. J. Geom. Anal. 4, (1994) 59-90.

17. LARChÉ, F. C. \& CAHN, J. W. Thermochemical equilibrium of multiphase solids under stress. Acta Metallurgica 26, (1978) 1579-1589.

18. LeO, P. H. \& HU, J. A continuum description of partially coherent interfaces. Continuum Mech. Thermodyn. 7, (1995) 39-56.

19. Matthews, J. W. Misfit dislocations. In Nabarro, F. R. N. (ed.), Dislocations in solids. NorthHolland, Amsterdam (1979).

20. Rockafellar, R. T. Convex analysis. Princeton Univ. Press, Princeton (1970).

21. VAn der Merwe, J. H. Crystal interfaces. Part I. Semi-infinite crystals. J. Appl. Phys. 34(1), (1963) $117-122$.

22. VAn Der Merwe, J. H. Crystal interfaces. Part II. Finite overgrowth. J. Appl. Phys. 34(1), (1963) 123127.

23. VAN DER Merwe, J. H. Interfacial misfit and bonding between oriented films and their substrates. In Francombe, M. H. \& S Ato, H. (eds), Single Crystal films, Pergamon Press, New York, (1964).

\section{Appendix: Korn's inequality}

We state and prove here a modified version of Korn's inequality. The proof follows Dautray \& Lions [8]. Recall that functions in $W$ are such that $\mathbf{u}(0,0)=0$. 
THEOREM If $\Omega$ is a bounded set with regular boundary, then there exists a positive constant $C$ such that

$$
\|\mathbf{E}\|_{L^{2}\left(\Omega, \mathbb{R}^{(2 \times 2)}\right)}^{2} \geqslant C\|\mathbf{u}\|_{W^{1,2}\left(\Omega, \mathbb{R}^{2}\right)}^{2}
$$

for any $\mathbf{u} \in W$, and with $\mathbf{E}$ the strain associated to $\mathbf{u}$.

Proof. The first step is to prove that $\|\mathbf{E}\|_{L^{2}\left(\Omega, \mathbb{R}^{(2 \times 2)}\right)}^{2}:=\int_{\Omega} \mathbf{E} \cdot \mathbf{E} \mathrm{d} x \mathrm{~d} y$ defines a norm on $W$, i.e. $\|\mathbf{E}\|_{L^{2}\left(\Omega, \mathbb{R}^{(2 \times 2)}\right)}=0 \Longrightarrow \mathbf{u}=0$. To see this, note first that if $\|\mathbf{E}\|_{L^{2}\left(\Omega, \mathbb{R}^{(2 \times 2)}\right)}=0$ then $\mathbf{E}=0$ a.e., and this in turn implies that $\mathbf{u}(x, y)=\mathbf{a}+b(y \mathbf{i}-x \mathbf{j})$, with $\mathbf{a}$ and $b$ constant. But since $\mathbf{u} \in W,(2.1)$ holds and $b=0$, while $\mathbf{a}=0$ is a consequence of $\mathbf{u}(0,0)=0$.

The second step, which shows that the norm $\|\mathbf{E}\|_{L^{2}\left(\Omega, \mathbb{R}^{(2 \times 2)}\right)}$ is equivalent to the $W^{1,2}\left(\Omega, \mathbb{R}^{2}\right)$ norm on $W$, is as in Dautray \& Lions [8]. 